\title{
In the news
}

\section{MELANOMA: SEEING RED}

People with pale skin and red hair have a higher risk of developing melanoma than those with other skin types. Now, a new study led by David Fisher of the Massachusetts General Hospital, Boston, USA, has suggested that there may be an underlying genetic basis for this increased risk that is independent of exposure to ultraviolet (UV) radiation.

To investigate the effects of pigmentation on melanoma development, the researchers generated three different mouse models - wild-type ('black') mice, which produced a high proportion of eumelanin; 'albino' mice, which produced no pigment; and 'red' mice, which produced a high proportion of pheomelanin. Before the researchers were able to expose the mice to UV radiation, around $50 \%$ of the red mice developed melanomas.

These results suggest that pheomelanin itself may increase melanoma risk: "There is something about the redhead genetic background that is behaving in a carcinogenic fashion. It means that shielding from UV would not be enough", said Dr Fisher (The New Zealand Herald, 2 Nov 2012). The group hopes that the results may lead to the development of more effective methods of melanoma prevention. However, Phillip Artemi of the Australasian College of Dermatologists warned that although the study was interesting, it has not yet been repeated and so, "we're really a long way from being able to say there's something else ... that could be triggering melanomas" ( $A B C$ News, 2 Nov 2012).

Julia Newton-Bishop, of the University of Leeds, UK, added that, despite these findings suggesting a genetic basis for the increased risk of melanoma in redheads, "protecting your skin in the sun is crucial" (Cancer Research UK, 1 Nov 2012). Indeed, as Dr Fisher commented, "about six out of seven melanomas will be cured if they are found early" (HuffingtonPost, 1 Nov 2012). 